\title{
EDITORIAL
}

\section{Using Discrete Choice Experiment to elicit patient preferences for osteoporosis drug treatments: where to from here?}

Tracey-Lea Laba

See related research by Hiligsmann et al., http://arthritis-research.com/content/16/1/R36

\begin{abstract}
Osteoporosis is a disease that increases skeletal fracture risk and places a significant health and economic burden on patients, families, and health systems. Many treatment options exist, but patient use is suboptimal, thus undermining the potential cost-effectiveness of treatments. In the previous issue of Arthritis Research \& Therapy, Hiligsmann and colleagues expanded the findings of previous studies to report, from a sample of 257 patients with osteoporosis, the preference to trade off clinical outcomes for the amenity provided by convenient dosing regimens. This editorial critiques the strengths and limitations of the methods, discusses the potential utility of patient treatment preferences, and suggests avenues for further research.
\end{abstract}

In the previous issue of Arthritis Research \& Therapy, Hiligsmann and colleagues explored the treatment preferences of patients with, or at risk of, osteoporosis [1]. Osteoporosis is a disease characterized by impaired bone quality, leading to increased fracture risk at multiple skeletal sites and significant morbidity and mortality [2]. The health and economic burden of osteoporosis to individuals, families, and health systems is substantial, and osteoporotic fractures represent $0.83 \%$ of the global burden of non-communicable diseases [3]. Currently, the prevalence of osteoporosis is higher in females, although men have higher fracture-related mortality rates [4]. The prevalence of osteoporosis and incidence of osteoporotic fractures are expected to increase, particularly as the population ages.

Correspondence: tlaba@georgeinstitute.org.au

The George Institute for Global Health, Level 10, King George V Building, 83-117 Missenden Road, Camperdown, NSW 2050, Australia
Preventing osteoporotic fractures, such as through the use of cost-effective drug treatment, is critical to sustainable health budgets worldwide. Several cost-effective treatment options are known to exist, offering patients a variety of dosing frequencies (for example, weekly and annually) and routes (for example, oral and intravenous). However, patients may for various reasons fail to take up or persist with osteoporotic treatments [5]. Understanding patient preferences for treatments can help clinicians prescribe treatments that patients like (and presumably will take) and can assist health-care funders identify treatments that are more likely to be cost-effective in practice.

In the study by Hiligsmann and colleagues [1], Discrete Choice Experiment (DCE) was used to elicit treatment preferences. DCE is a robust survey methodology that presents respondents with successive choices between hypothetical treatments. An underlying assumption is that choice is driven by trading between treatment attributes (for example, side effects and efficacy). When econometric modeling is used, the relative importance of attributes and the extent to which respondents trade between attributes of treatment decisions can be quantified [6]. DCE is used widely in health to address problems ranging from assessing medication adherence $[7,8]$ to predicting the uptake of government health policies [9].

The capacity of a DCE to accurately understand behavior is dependent on the choice of attributes posed to respondents. The attributes used by Hiligsmann and colleagues [1] include efficacy (that is, reduction of fracture risk), side effects, administration mode and frequency, and patient costs. Importantly, these attributes were determined though the analysis of qualitative data collected from patients with osteoporosis [10]. Furthermore, a 'no treatment' option was posed, thereby mimicking real-life choices. These methodological features strengthen the research findings. 
In light of the current literature assessing osteoporosis treatment preferences, the findings that patients prefer treatments offering higher efficacy, lower costs, and less frequent dosing regimens and that not all patients have the same preferences are not surprising. This study does, however, evaluate additional administration routes currently or soon to be available, the side effects deemed important by patients, and the perspectives of men - currently underrepresented in the literature yet predicted to contribute significantly to the total cost burden of osteoporosis [4]. Furthermore, the extent of efficacy and personal monetary cost that participants were willing to forego in exchange for desired dosing characteristics were quantified. For decisionmakers, this evidence demonstrates that maximizing treatment efficacy and safety is not unequivocally important to all patients, particularly if at the cost of less convenient administration attributes.

There are some caveats to these findings and thus room for further research. First, all DCEs are subject to hypothetical bias: what respondents say they would do may differ from what they actually do, especially when experiencing the consequence of a choice. Hypothetical bias is unavoidable in DCEs but can be minimized. Combining results with actual choice data (for example, real-world data capturing actual choices by patients) is one technique that needs further exploration in health.

Second, preferences were captured from consecutive patients attending one osteoporosis center and at one point in time. In addition to investigating the external validity of the findings, the temporal variations in preferences need to be better understood, particularly as patient preferences are likely to change over the course of treatment.

Finally, further work is needed to understand preferences in subgroups. For instance, the results comparing preferences of high- versus low-risk patients suggest that patients at high risk have an increased preference for medication with lower efficacy compared with low-risk patients; this is somewhat counterintuitive and needs further investigation. Research into the preferences of men and racial and ethnic minorities is needed given the rapidly increasing share of the osteoporosis disease burden projected in such populations [4].

In summary, Hiligsmann and colleagues have provided further evidence of the treatment preferences of patients with osteoporosis, highlighting the willingness of patients to trade off clinical outcomes for the amenity provided by convenient dosing regimens. The authors have good reasons to believe that this information will be of interest to clinicians and health-care decision-makers. However, given the suboptimal use of osteoporotic medications by patients, more work is needed to further understand how stated treatment preferences translate to real-world patient use of medications over time. Ultimately, this will better inform the interpretation of cost-effectiveness evidence for osteoporosis treatments and promote more effective and cost-effective treatment of this condition.

\section{Abbreviation}

DCE: Discrete Choice Experiment.

\section{Competing interests}

The author declares that she has no competing interests.

\section{Acknowledgments}

The author is funded by a National Health and Medical Research Council (NHMRC) Capacity Building Grant (57132) and would like to thank Stephen Jan and Jane Carland for proofreading the manuscript of this editorial.

Published: 10 Mar 2014

\section{References}

1. Hiligsmann M, Dellaert BG, Dirksen CD, van der Weijden T, Goemaere $S$, Reginster JY, Watson V, Boonen A: Patients' preferences for osteoporosis drug treatment: a discrete-choice experiment. Arthritis Res Ther 2014, 16:R36.

2. Rachner TD, Khosla S, Hofbauer LC: Osteoporosis: now and the future. Lancet 2011, 377:1276-1287.

3. Johnell $O$, Kanis JA: An estimate of the worldwide prevalence and disability associated with osteoporotic fractures. Osteoporosis Int 2006, 17:1726-1733.

4. Burge R, Dawson-Hughes B, Solomon DH, Wong JB, King A, Tosteson A: Incidence and economic burden of osteoporosis-related fractures in the United States, 2005-2025. J Bone Miner Res 2007, 22:465-475.

5. Gold DT: Understanding patient compliance and persistence with osteoporosis therapy. Drugs Aging 2011, 28:249-255.

6. Bridges JF, Hauber AB, Marshall D, Lloyd A, Prosser LA, Regier DA, Johnson FR, Mauskopf J: Conjoint analysis applications in health - a checklist: a report of the ISPOR Good Research Practices for Conjoint Analysis Task Force. Value Health 2011, 14:403-413.

7. Laba TL, Brien JA, Fransen M, Jan S: Patient preferences for adherence to treatment for osteoarthritis: the MEdication Decisions in Osteoarthritis Study (MEDOS). BMC Musculoskelet Disord 2013, 14:160.

8. Laba TL, Brien JA, Jan S: Understanding rational non-adherence to medications. A discrete choice experiment in a community sample in Australia. BMC Fam Pract 2012, 13:61.

9. Bridges JF, Dong L, Gallego G, Blauvelt BM, Joy SM, Pawlik TM: Prioritizing strategies for comprehensive liver cancer control in Asia: a conjoint analysis. BMC Health Serv Res 2012, 12:376.

10. Hiligsmann $M$, van Durme $C$, Geusens $P$, Dellaert BG, Dirksen $C D$, van der Weijden T, Reginster JY, Boonen A: Nominal group technique to select attributes for discrete choice experiments: an example for drug treatment choice in osteoporosis. Patient Prefer Adherence 2013, 7:133-139.

10.1186/ar4501

Cite this article as: Laba: Using Discrete Choice Experiment to elicit patient preferences for osteoporosis drug treatments: where to from here? Arthritis Research \& Therapy 2014, 16:106 